\title{
Formación en comunicación y dirección en algunas maestrías en gerencia de empresas
}

\author{
Luis Rodolfo Rojas Vera * \\ Elizabeth Arapé Copello**
}

\section{Resumen}

Este artículo reporta algunos resultados de investigación comparativa sobre los componentes de comunicación en Programas de Maestría en Dirección de Empresas en España y Venezuela. Hace énfasis en la relación entre la oferta contenida en los programas y las tendencias teóricas en la materia. El artículo muestra una visón interpretativa basada en datos cuantitativos y cualitativos. Ofrece reflexiones útiles para las Maestrías en Dirección de Empresas.

Palabras clave: Comunicación, dirección, administración, gerencia, maestrias, España, Venezuela.

\section{Communication, Direction and Formation in Certain Masters Degree Programs in Business Management in Spain and Venezuela}

\section{Abstract}

This paper reports on the results of a comparative study of the communicational components of certain masters degree programs in business management between two

\section{Recibido: 00-8-97 . Aceptado: 20-11-97}

* Dr. En Ciencias de la Información por la Universidad Autónoma de Barcelona (UAB), Profesor Titular de la Universidad del Zulla, acreditado en el programa de promoción al investigador (PPI), Investigador Responsable del Proyecto "Comunicación y Dirección”. Universidad del ZuliaCONDES. Autor para la correspondencia. Dirección: P.O.Box. 15.172 Maracaibo Venezuela / Email: luisrojas iamnet com; lurojas conicitve; telfax: +5861529724 .

** Estudios Doctorales en Ciencias de la Información (UAB), Documentalista del Proyecto 1915.95. 
countries: Spain and Venezuela. Emphasis is made on the relationship between the program contents offered and the theoretical tendencies of the courses. This article presents an interp retive vision based on qualitative and quantitative data. Thoughts on the utility of masters degree programs in business management are also offered

Key words: Communication, Direction, Administration, Masters degree programs, Management, Spain, Venezuela

\section{Introducción}

Este artículo parte del proyecto "Comunicación y Dirección" con el que se pretende explorar la importancia que se le asigna a la formación en Comunicación dentro de algunos Programas de Escuelas de Negocios. Específicamente trata de interpretar y valorar el impacto de la atención a la Comunicación en la formación de Directivos o Gerentes y sus estilos, en programas del tipo de Master in Business Administration o Master in Management. El artículo ofrece una revisión teórica del tema que contrasta luego con los resultados comparativos de las ofertas de estudio de varias Escuelas de Negocios de dos países.

La investigación parte de la importancia de la educación en comunicación para la formación de los directivos y el ejercicio de la dirección en las organizaciones. La idea surge por tres razones fundamentales: 1) la preponderancia de la comunicación en la sociedad y la empresa actual, caracterizada por el concepto de sociedad postindustrial o sociedad de la información, y cuyo impacto se extiende desde el campo de la tecnología de la comunicación hasta la importancia de la comunicación en la estrategia; 2) el impacto creciente de la ciencia y el arte de la comunicación en la dirección o management, como ruta del entendimiento entre las partes, como soporte de la negociación, la integración, el entendimiento y el avance; y 3) la concepción de las organizaciones empresariales como comunidades de intereses y por tanto comunidades comunicacionales, que se potencian o relentizan mediante la cultura de la comunicación en su más amplio sentido: desde lo humano a lo tecnológi$\mathrm{co}$, desde lo operativo a lo estratégico.

A los efectos de este trabajo entendemos por comunicación, organización y dirección lo siguiente: 1) comunicación: el intercambio progresivo de informaciones, sentimientos o saberes destinados tanto al conocimiento y las transformaciones como crecimiento común de todas las partes involucradas, comunicación como una fuerza intangible de la inteligencia empresarial; 2) organización: comunidad empresaria o agrupación humana encaminada hacia una misión aceptada en la comunidad de intereses gracias a la comunicación creciente; comunidad que puede nacer, crecer o morir gracias al impacto de la fuerza comunicacional; 3 ) dirección: el rumbo que toman las acciones de una organización empresaria, el sentido que proporciona coherencia en el hacia dónde vamos, la acción comunicativa 
hacia la transformación. Evidentemente, la interpretación de estos términos clave puede tan ser amplia como perspectivas sean posible. Resultaría difícil poner de acuerdo las múltiples matizaciones conceptuales que imperan tanto en el ambiente académico como en el empresarial. La dificultad crece con la fuerza del pensamiento sindicalizado que intenta imponer especificas visiones. Sin embargo, para obviar tal dificultad en el contexto de este artículo cuando nos estemos refiriendo a alguno de estos tres términos claves se entenderá el enfoque global que hemos planteado en este párrafo.

Dos ideas resultan interesantes de entrada: 1) sin comunicación no hay dirección, y esto se demuestra desde la acción de liderazgo hasta la acción de la comunicación corporativa, desde los procesos de trabajo hasta los impactos de las nuevas tecnologías de la información y la comunicación; $y, 2$ ) la dirección es metáfora de la cultura comunicacional, es decir: nuestro modo de entender la comunicación, y la interacción comunicativa humana y organizacional, puede determinar nuestros estilos directivos, aunque mucha veces se resalte el efecto de la cultura directiva sobre la comunicacional. En consecuencia, las implicaciones mutuas entre Comunicación y Dirección son diversas, multidimensionales y de alto impacto aunque por obvias no parezcan tan estudiadas.

Este trabajo se fundamenta en la importancia de la formación en comunicación tanto para la educación del directivo como para el ejercicio de éste dentro del desarrollo de la acción empresarial.
Se valora inicialmente a la comunicación como un factor clave del éxito en los dominios dirección y empresa (Herreros, 93; Soreson, 90; Rojas Vera, 94a, 95a; Rojas y Herreros 94).

A nivel teórico la importancia de la comunicación es un signo característico de la acción directiva y empresarial de nuestros días, $y$, como deciamos antes (Rojas Vera, 1994 b), la comunicación es un valor intangible que cobra cada día mayor importancia. Con la evolución de la sociedad agraria a la industrial se desplazó el centro de atención de la tierra a los procesos, luego con el ingreso a la sociedad informacional la atención se ha centrado en el intercambio del saber (Drucker, 1994; Páez, 1992).

Estas mismas evoluciones sociales han generado cambios en los paradigmas directivos y empresariales desplazándose desde estilos impositivos a formas basadas en la cultura de la negociación y comunicación, pasando por la época donde las finanzas, el márketing y las operaciones de producción se convertían en la explicación máxima. La cultura de la sociedad de información y de la comunicación es la matriz en la cual se desarrollan las organizaciones actuales y tal vez nos conduzca a plantearnos un reaprendizaje de la cuestión comunicacional para la dirección. Más que tecnológica la cuestión puede plantearse en términos de la hipótesis de reentender el entendimiento de la comunicación para dirigir la dirección. Una dirección clásica puede estar en crisis en un mundo regido por la comunicación. 
Revisión teórica: comunicación y dirección

En general existe un amplio soporte científico que explica las relaciones entre comunicación y dirección. Normalmente se habla de la influencia determinante de la dirección sobre la comunicación planteándose ésta ultima como instrumento de la primera. Parece imperar un enfoque que sitúa a la comunicación como instrumento de la dirección, que convierte a la comunicación en metáfora de la dirección. Por otra parte existe una valoración creciente de la importancia e impacto de la comunicación sobre la dirección y la gestión en la organización, idea que podría resumirse en el planteamiento poco reconocido de que la organización es producto de su cultura comunicacional. Ello revela la importancia del tema y su creciente atención científica. Las diversas contribuciones cientificas que se suscitan en esta línea dibujan un mapa conceptual en el que encontramos desde temas puntuales hasta generalistas ${ }^{\dagger}$.
Dentro de esta revisión destacan siete líneas de trabajo que captan nuestra especial atención por plantear un amplio e interesante abordaje de la cuestión comunicacional en la dirección de las organizaciones. Son líneas que explican la importancia de la comunicación en la dirección y que por tanto nos iluminan en la interpretación para analizar la atención prestada en la formación en Comunicación en los Programas de formación de Directivos que seleccionamos bajo la denominación de Programas de Maestría o de Máster.

\section{Nuevas Concepciones}

Fulk y Boyd (1991) nos hablan de las teorías emergentes para explicar el tema comunicacional en las organizaciones, valorando desde la cuestión tecnológica hasta el impacto de la comunicación humana. Lo importante de esta visión es el entendimiento de la comunicación como un problema realmente multidimensional y envolvente de la trama empresarial. Los problemas comunicaciona-

Entre estos se encuentran Comunicación Humana (Watzlawick et al, 1995) Comunicación Directiva (Rojas Vera, 1994a; Rojas Vera y Herreros, 1994); Comunicación Corporativa (Lessem, 1992; Herreros, 1993); Comunicación Total (Stewart, 1992); Comunicación Interna (Andreu, 1996); comunicación y creación para el desarrollo de equipos (Bucholz y Roth, 1987); Comunicación y creatividad para el cambio (Ford, 1996); Comunicación y creatividad como clave del pensamiento estratégico para las empresas del futuro (Murakami y Nishikami, 1993); Comunicación organizacional (Martínez, 1988); Comunicación y solución de problemas de trabajo (McCall, 1990); Comunicación y liderazgo (Adair, 1990; Bass, 1985; Bennis y Bust 1985; Bruman, 1992; Cohen y Bradford (1990); Comunicación y visión (Namaki-El, 1992; Rojas Vera, 1994. Comunicación y tecnologías de la información (Andreu et al., 1991; Comellá, 1994); Comunicación e inteligencia empresarial (Paéz, 1992; Rojas Vera, 1994b; Fayard, 1996); Comunicación y cambio de paradigma (Drucker, 1994; Sharp, 1988; Totfler, 1995; Nueno, 1996); y, entre otras tantas líneas de trabajo, Comunicación y programación neurolungüistica (Ribeiro, 1994). 
les dentro de las organizaciones en realidad entremezclan situaciones diversas y complejas en las que resulta dificil una separación entre efectos y causas de órbitas aparentemente distantes como lo humano o las nuevas tecnologías de la comunicación.

Desde esta perspectiva se critica la segmentación excesiva del tema. Tal segmentación puede conducirnos por caminos de interpretaciones parciales que nos pueden atrapar en visiones miopes sobre el tema-problema de la comunicación. Fulk y Boyd plantean la necesidad de enfoques amplios e interrelacionados para abordar el tema de las comunicaciones. Una visión e interpretación holística diff́cil de captar pero más integral que la clásica interpretación mecanicista y reduccionista del asunto comunicacional. Más allá de una visión instrumental de la comunicación plantean la necesidad de un abordaje interdisciplinario.

\section{La orientación principal}

Soreson, Savage y Orem (1990) luego de estudiar mas de 250 escuelas de negocios detectan tres grandes áreas de interés que direccionan la Orientación Principal del entendimiento y la acción comunicacional. Estas áreas son: 1) Business Communication, como el conocimiento y habilidades para transmitir personal y colectivamente la idea del negocio, especialmente en su dimensión estratégica; 2) Managerial Communication, como el conocimiento y habilidades para transmitir y coordinar el esfuerzo del trabajo especialmente en la órbitas interpersonales, y 3) Organizational Communication, como la consolidación de conoci- mientos y acciones comunicacionales que determina la agradabilidad, eficiencia, y efectividad de la cultura comunicacional, los climas comunicacionales y el progreso de la comunicación en la comunidad.

En esta línea Soreson, Savage y Orem insisten en la amplitud del término comunicación y llegan a concebirlo como el gran tejido unificador de las organizaciones indicando su misma acción directiva. Sin embargo, impera un sentido principalmente pragmático del asunto al centrar el enfoque en una interpretación de la comunicación como instrumento.

\section{La consultoría y la comunicación}

Shein (1990) nos habla del valor de la comunicación interpersonal para ayudar a las personas en las empresas. EI valor de la cultura comunicacional para la convivencia en la empresa entendida ésta en cierto modo como una comunidad de pensamientos, una comunidad de comunicaciones. De nuestra cultura comunicacional puede depender nuestro ejercicio de la dirección. Shein nos llama la atención sobre tres niveles en los cuales la comunicación juega un importante papel en la consultoría: los contenidos que se intercambian, los procesos comunicacionales, y la estructura o recurrencia de la comunicación. Esto, inclusive, puede estar siendo alimentado por las contribuciones de los avances en la programación neurolingüistica y la sicología cognitiva, como podrá verse más adelante.

Al hablar de consultoría y comunicación se pone el énfasis en el entendimiento interpersonal para la ayuda mutua de las partes como factor de convivencia 
organizacional. Más que de comunicación impositiva o unidireccional, que impera casi genéticamente dentro del mundo de la clásica cultura empresarial, se trata de explorar la cultura comunicacional para ayudar a mejorar la vida humana dentro de la organización.

Estas visiones pueden ser ampliadas con contribuciones como las de Old (1995) o Senge (1992) quienes nos plantean un reaprendizaje de la comunicación en la empresa moderna.

\section{Comunicación y tecnologias de la información}

La cuarta línea se refiere a la integración de recursos de tecnologías de la información y sistemas de información (SI/TI) a los problemas generales de la comunicación en la empresa, como una cuestión que nos orienta a la inteligencia comunicacional. Andreu et al (1991), Cornellá (1994) y Páez (1992) nos hablan de la integración de los $\mathrm{SI} / \mathrm{TI}$ al problema del pensamiento estratégico de la comunicación, es decir al problema del saber en la empresa. Es demasiada la complejidad, impacto y responsabilidad de la relación SI/TI para que no forme parte del pensamiento estratégico comunicacional de la organización, para que esté fuera de la inteligencia empresaria basada en los avances de la cultura de la comunicación.

Otros autores como Fayard (1996) destacan la importancia de coordinar las entidades $\mathrm{S} / \mathrm{TI}$ desde un alto pensamiento estratégico comunicacional. Sin un pensamiento comunicacional integrador estratégico los $\mathrm{SI} / \mathrm{TI}$ no pasarán de simples recursos. El valor estratégico del triángulo $\mathrm{SI} / \mathrm{TI}$-Estrategia Empresarial-
Cultura Comunicacional es una de las ventajas que están experimentando las empresas inteligentes que valoran más la estrategia de los intangibles que el peso de la tecnología mecánica.

\section{Comunicación y creatividad}

La quinta línea es la que nos ofrecen autores como Murakami y Nishikami (1993) para relacionar directamente a la comunicación con la creatividad del pensamiento estratégico como factor determinante del éxito en las empresas de los próximos años. La comunicación y la creatividad, y su dominio en el más amplio sentido, será el más importante de los valores añadidos para las empresas que tengan resueltos los parámetros tecnologicos característicos de la sociedad industrial. En la sociedad de la información, y mirando más allá de ella, las reglas del juego no serán las tecnológicas sino la creatividad de los nuevos pensamientos y su rápida y efectiva comunicación. Por tanto, ello plantea un déficit conceptual y un reto directivo.

Murakami y Nishikami insisten en la necesaria creatividad del pensamiento para entender las claves de la moderna sociedad comunicacional o postinforma. cional. Más allá de la tecnología y sus efectos sobre la organización el nuevo reto directivo está orientado al entendimiento de la organización como comunidad creativa. Los nuevos grupos o tipos de trabajadores pueden ser solo tres: los creadores de soluciones o emprendedores, los impulsadores o socios, y los bloquedores o creativamente minusválidos. Parte del éxito de la creatividad está en la capacidad del pensamiento creativo para 
generar alianzas con los impulsadores y esquivar a los bloqueadores, en la fuerza de la cultura comunicacional para vender una idea, lograr apoyo y conseguir seguidores, en el reconocimiento de la comunicación para la integración de la acción creativa y transformadora. Reconocer la acción directiva de la comunicación desde la fuerza creativa y sobre la misma dirección, puede llevarnos a reconocer que la empresa es producto de su comunicación, que la empresa es metátora de su cultura comunicacional.

\section{La comunicación humana}

Watzlawick et al (1995) desde la Escuela de Palo Alto han puesto el énfasis en la cultura comunicacional humana como evidencia del entendimiento en las organizaciones. La comunicación, en tanto reflejo del pensamiento, expresa la salud personal e interpersonal de los actuantes como integrantes de organizaciones. Desde esta perspectiva la comprensión de la comunicación abarcaría tres grande órbitas de utilidad para el entendimiento comunicacional dentro de las organizaciones: 1) la semántica: referida a la capacidad de traducción y compartición de los significados, a la retórica, a las metáforas, como contenido de lo común en la comunicación; 2) la sintáctica: como la organización estructural de los significados, el efecto de la redundancia, los ruidos, y las repeticiones para el proceso de intercambio y entendimiento, $y, 3$ ) la pragmática: como la traducción conductual de los efectos comunicacionales, como el efecto transformacional logrado. En cierto sentido, tanto a nivel personal, como grupal o corporativo, el plano de la semántica equivaldría al entendimiento filosófico de las ideas comunicadas; la sintáctica a la lógica o proceso de intercambio de la información o significados; y la pragmática a la conducta o acción personal, grupal o empresarial expresada. En realidad, pues, comunicación implica al menos estos tres niveles complejos que nos hablan de perspectivas interconectadas.

Los tres planos resultan interesantes porque nos revelan niveles de acción y acuerdos. Podemos estar de acuerdo en los contenidos pero no en los procesos; podemos tener acierto en los contenidos y las formas comunicadas pero no en las acciones transformacionales logradas como producto de tal comunicación.

\section{Comunicación tranformacional}

Autores como Habermas (1982, 1991) nos han presentado la necesidad de reentender la comunicación más allá de cualquier función crematística, y plantearla como una de las principales vías para el entendimiento, y la salvación. Baudrillard (1992, 1994) nos habla de la desilusión de la comunicación como el conjunto de las paradojas del desencuentro en el encuentro. Nos encontramos en un mundo que no sabemos si está más comunicado que antes aunque tengamos más acceso a la comunicación, donde pareciera que todo, como la comunicación, fuese mas virtual que real y donde la intangibilidad de lo virtual comienza a dirigir a lo real como explicación postmoderna.

Las posibilidades comunicacionales en la sociedad y en la empresa han crecido exponencialmente, pero recono- 
cemos que hay un saldo negativo en el entendimiento humano, que hay una deuda con el hombre, que la comunicación pareciera tener un sentido inverso, virtual, y hasta descomunicante. La intimidad y trascendencia de los problemas lo revela aunque caigamos en la trampa de no aceptarlo.

En realidad esta línea nos plantea la reinterpretación de la comunicación en su sentido ontológico. Más allá de la incomprensión de la comunicación, tal vez por la preponderancia de las visiones utilitarias, es necesario plantear una Comunicación Transformacional ${ }^{2}$ (Rojas Vera, 1995) que ayude al hombre a transformar el mismo entendimiento del sentido de la comunicación, reto que afecta a las relaciones entre comunicación y dirección ya que cabría la hipótesis de que la comunicación podría dirigir a la dirección. A mayor comunicación de calidad, a mayor calidad de comunicación, a mayor entendimiento humano, a mayor entendimiento instantáneo por desarrollo de la cultura comunicacional, posiblemente menor sea la necesidad de direccionalidad, menor efecto directivo tendrá la dirección no comunicacional, y mayor impacto directivo tendrá la comunicación intangible. A mayor entendimiento comunicacional menor dependencia de la dirección y mayor necesidad de reentender y reinterpretar a la comunicación.

En general podriamos entender esta línea como el planteamiento de los valores postmodernos para reinterpretar el problema de la comunicación en la organización (Gillespie y Meyer, 1996; Llano, 1994; y Rojas Vera, 1996). Más allá de los problemas técnicos de la comunicación la cuestión es fundamentalmente de naturaleza filosófica, ideológica. ¿Podemos creer en la verdadera comunicación como fuerza recuperadora y reconstructora de un modelo empresarial en crisis?

\section{El balance}

¿Qué tenemos hasta ahora? La revisión teórica nos muestra una amplia discusión del tema de la comunicación en la dirección, llamando la atención la apertura de nuevos horizontes. Ahora resulta necesario contrastar tales avances teóricos con la formación que se ofrece en algunas Escuelas de Negocio. Hemos orientado nuestra búsqueda sobre la oferta que dentro de los programas de

2 El Concepto Comunicación Transformacional fue desarrollado como uno de los pilares teóricos del Seminario "Comunicación y Dirección" del Doctorado en Ciencias de la Comunicación, y del Seminario "Comunicación y Empresa" del Master in European Business Communication, impartidos ambos por los autores como Profesores Visitantes en la Universidad Autónoma de Barcelona en el año académico 1995-1996, y que posteriormente se transformaría en el "Seminario de Comunicación Transformacional y Empowerment" impartido por el Dr. Rojas Vera en la misma universidad en junio de 1997 como Profesor Invitado de la Red Com Strategy / ICIIntercampus. 
tipo Máster tenemos de materias, asignaturas o seminarios relacionados directamente con la comunicación. Centramos la atención en cuatro dimensiones ${ }^{3}$ fundamentales que se desprenden de los avances teóricos en el campo. Estas cuatro dimensiones revelan la formación en comunicación a través: 1) Comunicación Organizacional y/o Corporativa, 2) Comunicación Interpersonal y Liderazgo, 3) Comunicación Tecnológica SI/TI, y 4) Nuevos Horizontes Comunicacionales.

\section{El proceso de investigación}

El trabajo de campo de esta investigación se circunscribe a una revisión en profundidad de una muestra de 14 Programas de Máster ${ }^{4}$ como programas de formación académica. En tanto se intenta contrastar los componentes orientados a la comunicación se han seleccionado: 10 Master in Business Administration (MBAs) y Máster en Dirección (management) y 4 Máster en Comunicación (MCs): La mitad de cada uno de los primeros de un país latinoamericano (Venezuela) y la otra de un país europeo (España). La mayoría de los MCs resultaron ser europeos. La mayoría de los MBAs tienen más de quince años de funcionamiento y sólo dos ellos tienen continuación directa hacia programas doctorales en Dirección de Empresas. Los $4 \mathrm{MCs}$ son de reciente creación y todos muestran un fuerte interés por el tema directivo. Los MCs se tomaron sólo como fuente de contrastación e información.
Para la selección de la muestra de los 10 Programas, a partir de una lista mayor, nos hemos basado en criterios cuantitativos y cualitativos atendiendo a: 1) el ranking de las escuelas en los paises respectivos; 2) el impacto y prestigio de sus instituciones valorado en relaciones con la investigación, publicaciones y relaciones con el entorno; 3) los contactos personales que permitieran confrontar aspectos cualitativos en la interpretación de resultados. La mayor parte de la información en Venezuela se recogió y contrastó en el primer semestre de 1995. Durante el resto de 1995 y el primer semestre de 1996, se recogió y procesó la información de España.

Los procedimientos de trabajo abarcaron tres ejes de acción: 1) Revisión e interpretación teórica de: a) el sentido de la formación en los MBA como laboratorios de formación de directivos, yb) la importancia del componente comunicación en tales programas, 2) Revisión e interpretación del contenido de las ofertas de tales programas y en especial de: a) su filosofia y b) el contenido de las asignaturas, y 3) contrastación interpretativa con profesores y responsables de algunos de los programas sobre: a) la experiencia de su escuela y b) las tendencias y perspectivas en el tema.

En general para conformar el marco teórico que ayudara a interpretar el sentido de la formación en comunicación dentro de los MBA nos apoyamos en autores como Ford (1996), McKenna, Cotton y Van Auken (1995), Roth y Senge

4 En ocasión del Año Sabático como Profesor Visitante en la Universidad Autónoma de BarceIona. 
(1996) Saini y Bhatia (1996), Soreson et. al (1990) Sebora, Hartman y Tower (1995), Sinha (1996) Rojas Vera (1995a, b; 1996). Los programas MCs se tomaron por tres razones: 1) la importancia que le asignaban a la formación directiva, 2) la posibilidad de contrastar la forma de aprendizaje con los MBA, y 3) un grupo significativo de profesores de MBA trabajan en ambos tipos de programas.

Para recoger la información de campo se elaboraron fichas de casos, revisión de programas ofertados, y entrevistas con profesores. Tomamos en cuenta un componente cuantitativo para dimensionar y contabilizar las materias relacionadas con comunicación y un componente cualitativo para interpretar la visión sobre los contenidos de esa oferta y la congruencia entre lo que se oferta y lo que dice entender de la comunicación.

\section{Algunos resultados}

Luego de efectuar una interpretación basándonos en el análisis de contenido de la documentación académica de los programas y contrastada en algunos casas con entrevistas personales con responsables académicos de los programas, llegamos a algunos resultados que se sintetizan a continuación.

\section{Visión / Misión}

Los MBAs coinciden en general con una misión declarada que se resume en la idea de "Desarrollar acciones comprometidas con la formación integral de lideres $y$ directivos de empresas y organizaciones." Sin embargo, existen dos visiones básicas que pueden orientar tal misión: por una parte una visión humanis- ta del ejercicio de la dirección, y por la otra una visión pragmática del asunto. Las visiones no son excluyentes y una combinación de ambas se integra en cada escuela. Desde la perspectiva humanista prima el sentido del hombre, la valores éticos y la responsabilidad social de la dirección; la comunicación está centrada en la persona, tiene un sentido de entendimiento y de desarrollo de la comunidad humana integrada en la organización y parece asociarse a aciones liberadoras. Desde la perspectiva pragmática se privilegia la eficiencia, la productividad y la administración de los recursos. La comunicación está centrada en el control y tiene un sentido instrumental, de mando unidireccional que pareciera ser principalmente crematística.

En realidad creemos que son dos polos de influencia que se entrecruzan y se combinan, y que con muchísima dificultad se podría estar drásticamente en algunos de los extremos sin ninguna influencia del otro.

Otro dato interesante resulta la distancia que se puede verificar entre lo que declara un Programa en su documentación escrita y lo que se traduce en el ejercicio de sus estilos. Hay programas que se declaran humanistas y actúan guiados por un pragmatismo exacerbado; hay otro segmento de programas que a pesar de no evidenciar su vocación humanista y declararse pragmáticos asignan una importantísima atención a los valores humanos. Es posible que estas diferencias se deban a: 1) problemas en las acciones comunicativas de los programas que afecten a los contenidos, los estilos y sus estructuras comunicacionales, o 2) a in- 
tencionalidades comunicacionales para adecuarse a sus mercados o entornos.

Los MCs declaran una misión relacionada con la formación de expertos en las Ciencias de la Comunicación y su aplicación social. Aunque se declara una visión humanista del tema, la orientación de sus acciones se encamina principalmente a un entendimiento de la comunicación como cuestión instrumental de la dirección de empresas. Programas como Máster en Comunicación Corporativa, Máster en Gestión de la Comunicación y Master in Business Communication podrían estar más cerca de una visión pragmática de la comunicación, que de una búsqueda del sentido humano de la comunicación centrado en la persona.

Visiones más amplias de la comunicación como en un Máster en Ciencias de la Comunicación podrían ofrecer una formación integral en torno a la comunicación de gran impacto para la dirección de las organizaciones. Aunque no llegasen a profundizar en los dominios de aplicaciones técnicas que proporcionan los máster pragmáticos, si podrian ofrecer a las organizaciones una reinterpretación filosófica y redirección del problema comunicación en la comunidades humanas, reconociendo a la comunicación precisamente como la zona donde acusamos gran parte de las patologias personales, interpersonales, grupales, organizacionales y corporativas.

\section{Los Perfiles Profesionales}

Para establecer los perfiles se fueron seleccionando un grupo de palabras clave que componen el léxico de un ambiente de cultura de escuelas de negocios con el cual ya se está familiarizado.
Se trataba de establecer un grupo de palabras claves que permitieran dibujar el perfil percibido del profesional a graduar. Luego se contabilizó su frecuencia de uso en los documentos en los que se habla del perfil buscado y en los que se reseña - registra los contenidos de las asignaturas a impartir en los programas. Para todas las escuelas se utilizó el mismo tipo de documentación: el material comunicacional o folleto oficial que se utiliza para vender el programa y que sive a los interesados para entender en profundidad su pasaporte al éxito. Diríamos que es la base comunicacional del contrato entre el oferente y adquiriente.

Inicialmente identificamos más de 100 palabras clave o descriptores de interés sobre la imagen o perfil del profesional. Algunas de estas palabras hablaban de la importancia de la comunicación. Luego se agruparon progresivamente en conceptos integrales que pudieran resumir a varios, bien por su semejanza o su valor de inclusión. Sencillamente se valoró si el programa ofertado hacía o no hincapié en determinados valores. Los valores predominantes y su frecuencia de uso en los diferentes Másters se muestran en la Tabla № 1 de Valores Declarado.

Una conclusión preliminar, en contra de lo que esperábamos, es que el valor comunicación tiene un peso relativamente bajo en la atención declarada tanto en los perfiles (20-25\%) como en los contenidos propuestos de las asignaturas. En la filosofía de los programas (visión / misión), en los contenidos generales de los programas (asignaturas ofertadas), en su imagen de venta, y en la interpretación de las entrevistas en profundidad, pareciera que la educación en comunica- 
Tabla 1

Valores Declarados en los

Programas de Gerencla de Empresas

Valores predominantes

$\%$ acuerdo en los MBAs

Venezuela

España

01. Líderes, Directivos

25

75

02. Gerentes, Managers

75

25

03. Negociadores Internacionales

25

100

04. Eticos-Humanos

50

75

05. Responsab. Social

75

75

06. Calidad, Productividad

75

50

07. Habilidades Administrativas

75

50

08. Habilidades Comunicacionales

20

25

09. Entasis Funcional / Especializante

100

75

10. Énfasis Generalista

25

75

11. Vocación Competitiva

100

100

12. Vocación Cooperativa

25

100

13. Énfasis en las relaciones con el entomo

25

75

14. Énfasis centrado en la organización

75

50

15. Capacitación Técnica

75

50

16. Desarrollo Humano y Personal

25

75

17. Pasaporte al éxito

25

100

18. Superación protesional

100

50

19. Nuevos horizontes

50

100

20. Independencia

50

75

Fuente: Elaboración propia

ción, el dominio de sus habilidades y técnicas, su dominio como arte y ciencia, y su valoración para el entendimiento global, fuera un tema o eje de formación prioritario. Pareciera que su atención estuviera cubierta por dos vías: 1) la educación formal mediante los contenidos pedagógicos estructurados en las asignaturas oficiales, y 2) la educación informal me- diante una especie de sociabilización en el máster o el autoaprendizaje del tejido de la vida del campo directivo marcado por valores como la competencia, el entendimiento y la negociación, la convivencia, el liderazgo, o el éxito, entre otros tantos.

En varias ocasiones algunos entrevistados argumentaban que la educación 
en comunicación se lograba de modo indirecto mediante el conjunto de diversas asignaturas, aún cuando estas no declararan tal cosa. Los entrevistados se basaban en el razonamiento de que en todas las asignaturas hay algo de comunicación.

\section{Las Asignaturas vinculadas} a la Comunicación:

Por lo general las materias o asignaturas que directamente se asocian con comunicación son del estilo, por ejemplo, de Comunicación Corporativa o Relaciones Interpersonales. Sin embargo, la lectura interpretativa, las entrevistas y los avances teóricos en la materia (Fulk y Boyd, 1991; Sorenson et. al 1990; Paéz, 1992) nos llevan a pensar en al menos cuatro grandes órbitas de materias relacionadas con la comunicación. Estas orbitas son: 1) la comunicación a nivel corporativo u organizacional, 2) la comunicación a nivel personal e interpersonal, 3) la comunicación en su dimensión tecnológica, y 4) las discusiones de nuevas fronteras comunicacionales.

En la Tabla № 2, titulada Asignaturas Relacionadas con Comunicación, mostramos el porcentaje de asignaturas que cada una de las escuelas o programas MBA dedica al tema de comunicación y que puedan ser incluidas dentro de estas cuatro categorías.

\section{Conclusiones}

De los resultados anteriores concluimos lo siguiente:

1) El porcentaje de las asignaturas relacionadas ampliamente con comunicación está entre el 20 y el $35 \%$ del total de asignaturas que componen los programa estudiados, siendo la oferta promedio del $26 \%$,

2) Del conjunto de asignaturas vinculadas a la comunicación: a) un promedio del $36.5 \%$ se orientan a temas globa-

Tabla N"2

Asignaturas Relacionadas con Comunicación en Programas de Gerencia de Empresas

\begin{tabular}{lcccccccccccc}
\hline & \multicolumn{1}{c}{ Venezuela } & \multicolumn{1}{c}{ España } \\
\hline Programas MBA No & 1 & 2 & 3 & 4 & 5 & 1 & 2 & 3 & 4 & 5 \\
$\begin{array}{l}\text { \% de las asignaturas relacio- } \\
\text { nadas con comunicación }\end{array}$ & 25 & 20 & 20 & 30 & 35 & 35 & 20 & 20 & 35 & 20 \\
$\begin{array}{l}\text { Orbita de las asignaturas: \% } \\
\text { s/ materias de comunicación }\end{array}$ & 40 & 40 & 30 & 40 & 40 & 40 & 35 & 35 & 35 & 30 \\
Corporativa/Organizacional & 40 & 40 & 30 & 40 & 40 & 40 & 35 & 35 & 35 & 30 \\
Liderazgo/Interpersonal & 40 & 40 & 40 & 35 & 35 & 40 & 35 & 35 & 40 & 45 \\
Tlisi & 20 & 20 & 30 & 15 & 20 & 20 & 30 & 30 & 15 & 25 \\
Nvos. Horizontes Comunicac & & & & & 05 & & & & & 10 \\
\hline
\end{tabular}

Fuente: Elaboración propia 
les como lo corporativo o la dimensión organizacional, siendo su banda del 30 al $40 \%$, b) Un promedio del $38,5 \%$ se orientan a temas personales como el liderazgo - la comunicación interpersonal, siendo su banda del 35 al $45 \%$; c) Un promedio del $22,5 \%$ se orienta a temas tecnológicos como Sistemas de Información o Tecnologías de la Información, siendo su banda del 15 al $30 \%$ y d) un promedio del $1,5 \%$ se orienta a temáticas de nuevas interpretaciones de la comunicación, como interpretaciones inter o multidisciplinarias, siendo su banda del 05 al $10 \%$.

Aquí pudiera incluirse la interpretación de la empresa como organización postindustrial y sus tendencias en la sociedad informacional. Pueden ser interesantes las discusiones originadas desde asignaturas de $\mathrm{SI} / \mathrm{Tl}$ o las exploraciones $\mathrm{e}$ interpretaciones de las organizaciones desde los "Open Spaces" que tienen algunos Programas para la libre discusión y revisión de las nuevas tendencias. Esta búsqueda de nuevas interpretaciones no constituyen una temática frecuente ni esperada en las ofertas del Máster tipo MBA pero podemos interpretarla como transformaciones emergentes en la visión de algunos entrevistados.

3) Cerca del $40 \%$ del esfuerzo en educación comunicacional se centra en las personas como líderes, directivos, gerentes o managers.

4) Los dos programas que incluían asignaturas 0 actividades formales de reflexión sobre las tendencias de la comunicación son dos de los tres que alcanzaron la mayor cuota de asignaturas relacionadas con comunicación: un $35 \%$.

5) En contraste, los cuatro programas que dedicaron mayor atención a ma- terias relacionadas con $\mathrm{SI} / \mathrm{TI}$ fueron los que dedicaron el más bajo porcentaje en materias generales de comunicación: $20 \%$.

6) Al contrastar las visiones humanistas y pragmáticas que se desprendían de la documentación de los programas se pudo constatar que sólo la mitad de los que se autodefinian humanistas coincidian con altos porcentajes de materias de comunicación.

7) La atención a nuevas interpretaciones de la comunicación se dio sólo en programas de declarada vocación humanista y uno de ellos con prolongación hacia doctorado.

8) El $80 \%$ de los programas ha sufrido una reorganización en los últimos cinco años y el actual porcentaje de materias relacionadas con comunicación ha sido producto de incrementos en tales reorganizaciones. Sin embargo se declaran apegados a una visión economicista o administrativa de la organización.

9) Los programas que menos han cambiado en los últimos cinco años argumentan su estado actual inspirados en lo pragmático del Know How de la dirección o en la lentitud de los cambios en las organizaciones a las que pertenecen.

Hacia el final de la revisión y el seguimiento de varios programas a lo largo de varios meses nos encontramos básicamente con lo siguiente: 1) Se reconoce la importancia creciente de la comunicación en el mundo empresarial y por tanto para la formación del Directivo, 2) Prevalece una visión y uso instrumental de la comunicación en la formación de directivo, 3) Prevalece una visión parcial y limitada sobre la comunicación asociada más con la transmisión de información 
que con la creación de comunidad de pensamiento, 4) La explicación de la comunicación se sigue haciendo desde la perspectiva del management industrial, 5) Los avances teóricos en materia de ciencias de la comunicación y su aplicación al management parecen ofrecer un gran horizonte de posibilidades que apenas comienza a ser explorado por algunos MBA, $y, 6$ ) Parece emergente una reconversión en los Programas de Formación de Directivos gracias al impacto del reentendimiento de la comunicación, principalmente desde dos ejes: la comunicación corporativa y las tecnologías de la comunicación. La valoración de la TI/S| con el pensamiento estratégico comunicacional y la valoración de la comunicación humana parecieran ser, dentro del campo comunicacional, las áreas problemas de más alto interés para los Máster en Dirección o Administración de Empresas.

Finalmente, no se pudo establecer un claro paralelismo entre los desarrollos teóricos en el campo de las ciencias de la comunicación descritos en la revisión bibliográfica, y su incorporación a progra- mas de Dirección de Empresas. La hipótesis tácita de que los Master en Gerencia estaban incluyendo sustanciales contribuciones desde las ciencias de la comunicación no se pudo verificar. Si se comprobó una especie de distancia entre lo declarado o anunciado en los prospectos oficiales y la interpretación de algunos entrevistados respecto a la valoración de la comunicación. Se sospecha que definitivamente los esfuerzos científicos en materia de comunicación y gerencia siguen por caminos separados, y que el pensamiento en los Master de Dirección consultados sigue siendo altamente pragmático. Por último, pareciera experimentarse una especie de déficit conceptual para entender, desde el modelo tradicional de gerencia, las nuevas realidades comunicacionales, lo que podría estar revelando una suerte de incultura comunicacional de la gerencia en la era de las comunicaciones ${ }^{5}$.

\section{Referencias bibliográficas}
Adair, John (1990) Líderes, No Jefes Legis, Fondo Edit. Bogota.

Los autores agradecen la cooperación prestada por los Profesores Dr. Joan Mundet-Hiern, del Departamento de Organización de Empresas de la Universidad Politécnica de Catalunya; Dr. Mario Herreros, del Doctorado en Comunicación de la Universidad Autónoma de Barcelona; Dr. Piere-Marie Fayard, de la Universitè de Poitiers; Dr. Gustavo Martin de la Universidad Central de Venezuela; Dr. Ricardo Haye de la Universidad de Comahue, Argentina; Prof. Cristina Ribas de la Universitat Pompeu Fabra, Prof. Luis Guadarrama de la Universidad de Toluca; y Dr. Savelly, de Amtserdam, entre otros. Especial agredecimiento a los alumnos de los Seminarios "Comunicación y Dirección" y "Comunicación y Empresa", 1995-96 y "Comunicación Transformacional y Empowement" 1997, impartido por los autores en la Universidad Autónoma de Barcelona. Los autores expresan su gratitud a las bibliotecarias de la Facultad de Ciencias de la Comunicación de la Universidad Autónoma de Barcelona por el apoyo linformacional internacional. 
Andreu, Ricart et. al. (1991) Estrategia y Sistemas de Información, McGraw Hill, Barcelona

Andreu Pinillos, Alberto (1996) "Comunicación interna, un paseo por el tiempo". Harvard Deusto Business Rev.70, (1) 48-63.

Barker, James R. ; y Cheney, George.(1994) "The Concept and the Practices of Discipline in Contemporary organizational life". Communication Monographis $61(3) 20-43$

Baudrillard, Jean (1992) La llusión del Fin, Anagrama, Barcelona

Baudrillard, Jean (1994) Las Estrategias Fatales, Anagrama, Barcelona

Bennis Warren y Burt Nanus (1985) Líderes, Edit. Norma, Bogotá

Buchkolz, S. y T. Roth (1987) Creating the Higt-Performance Team, John Wifley, N.Y.

Bruman, Alan (1992) Charisma and Leadership in Organizations, Sage Pub. London

Cohen, Alan y David Bradford (1990) Influence without Autority, John Willey, N.Y.

Comellá, Alfons (1994) Los recursos de la información, McGraw Hill, Barcelona Drucker, Peter (1994) 'The Age of Social Tranformation", Atlantic Monthly, 5 (247) 53-80

Fayard, Pierre-Marie (1996) "El Arte Estratégico: Horizontes de la Información y la Comunicación "Rev. Quark $n^{2} 2$, 1996, 33-48

Ford, Cameron (1996) "The role of creative action in organizational learning and change". Journal of Organizational Change Management 9 (1) 54-62

Fulk J. y B. Boyd (1991) "Emerging Theories of Communication and Organization", Joumal of Management, 17 (2) 407-446
Gillespie, Janet y Gordon Meyer (1996) "Gender, voice, electronic communication and postmodern values", Journal of Organizational Change Management, 6 (2) 29-44

Habermas, Jürgen, (1982) Conocimiento e Interés, Taurus, Madrid

Habermas, Jürgen (1991) Conciencia Moral y Acción Comunicativa, Peninsula, Barcelona

Herreros Mario (1993) En Torno a la Comunicación Corporativa", Rev. Area 5-CAP, Univ. Complutense de Madrid, 6 (2) 24-32

Llano, Carlos (1994) El Postmodernismo en la Empresa, McGraw Hill, México

Lessem, Ronnie (1992) Gestión de la Cultura Corporativa, Díaz de Santos, Madrid.

Martínez, Alberto y Abraham Nosnick (1988) Comunicación Organizacional, Trillas, México

McCall, lan y John Cousins (1990) Communication Problem Solving, John Wiley, U.K.

McKenna, John F.; Chester C. Cotton and Stuart Van Auken (1995) Busines School Emphasis on Teaching, Research and Servive to Industry. Journal of Organizational Change Management. 8 (2) 3-16.

Murakami, T. y T. Nishikami (1993) Estrategia para la Creación, Panorama, México

Namaki, El (1992) "Creating a Corporative Visión" Long Range Planning Rev. 25 (6) $25-29$

Nueno, Pedro (1996) Evolución de los Conceptos de Management, Barcelona Management Review, 1, Jan-apr, 1996, 73-83

Old, Dianna (1995). Consulting for real transformation, sustanablity, and organic form, Journal of Organizational Change Management, 8 (3) 6-17 
Páez, Iraset (1992) Gestion de la Inteligencia: Aprendizaje tecnológico y Modernización del Trabajo Informacional, IDESCO, USB, Caracas.

Ribeiro, Lair (1994) La Comunicación Eficaz, Urano, Barcelona

Rojas Vera, Luis R. (1994 b) El Cerente Paradigmas y Retos para su Formación, Rev. Encuentro Educacional, 1( 1) 1-28

Rojas Vera, Luis A(1994 a) La Comunicación Factor clave del éxito en los negocios Rev. Cuestiones Publicitarias, Univ. de Sevilla, 3 (2) 58-72

Rojas Vera, Luis y Mario Herreros (1994) Comunicación y Visión Directiva: Asignatura Clave. Rev. del Master en Comunicación Corporativa, Univ. Complutense de Madrid, 1 (2) 191-204

Rojas Vera, Luis Rodolto, y Elizabeth Arape (1995a) Empresa y Comunicación: Hacia una teoría del Postmanagement, Rev. RCS, FACES, LUZ 2 (3)

Rojas Vera, Luis Rodolio (1995b) La Comunicación Transformacional: Comunicación y Dírección, Materiales mimeografeados preparados para el Seminario homónimo impartido por el autor en el Doctorado de Cienclas de la Comunicación, Universidad Autónoma de Barcelona, 1995-96

Rojas Vera, Luis (1996) La Postcomunicación, Rev. Quack, 2 (2) Barcelona

Roth, George y Peter Senge (1996) From theory to practice: research territorry, processes and structure at an organlzational learning center,
Journal of Organizational Change Management, 9 (1) 92-106

Saini. J.S., y B.S. Bhatia (1995) Impact of Entrepreneurship Development Programs, The Journal of Entrepreneuriship, 5 (1) 65-88

Sinha, T.N. Human Factos in Entreprenuriship Effectiveness, The Journal of Entrepreneuship, 5 (1) 23- 64

Sebora, Terrence, Alan Hartman, y Burck Tower Education Entrepreneurship on innovation: Implication from an Investigation of Small Business Journal of Business \& Entrepreneurship, 7 (2) 1-13

Senge, Peter (1992) La Quinta Disciplina, Granica, México.

Shein, Edgar (1990) Consultoria de Procesos, Addison -Wesley Ib. México

Stewart, David (1992) Initiating Reform in Total Communication Program, Journal of Special Education 1( 26) 68-84

Sharp, Nancy et. al. Communication Research: The Challenge of Information Age, Siracuse Press Univ. N.Y.

Soreson, R., G. Savage y E. Orem (1990) A Profile of Communication Facultiy neens in Business Scholls and Colleges, Communication Education, 2 (39) $148-160$

Toffler, Alvin (1995) La Empresa Flexible, Plaza y Janes, Barcelona

Watzlawick, J., B. Bavelas y D. Jackson (1995) Teoría de la Comunicación Humana, Edit. Herder, Barcelona 